\title{
Trigeminal neurofibroma in the infratemporal fossa arising from the inferior alveolar nerve: A case report
}

\author{
TAKURO INOUE ${ }^{1}$, MOSTAFA ELASKARY ${ }^{2}$, AYAKO SHIMA ${ }^{1}$, \\ HISAO HIRAI $^{1}$, FUMIO SUZUKI ${ }^{1}$ and MASAYUKI MATSUDA ${ }^{1}$ \\ ${ }^{1}$ Department of Neurosurgery, Subarukai Kotoh Kinen Hospital, Higashiohmi, Shiga 527-0134, \\ Japan; ${ }^{2}$ Department of Neurosurgery, University of Alexandria, Alexandria 21526, Egypt
}

Received June 27, 2017; Accepted September 4, 2017

DOI: $10.3892 / \mathrm{mco} .2017 .1410$

\begin{abstract}
Solitary neurofibromas arising from cranial nerves are rare, and those arising from the peripheral divisions of the trigeminal nerve are even rarer. Although infratemporal fossa (ITF) masses are challenging to remove, certain approaches are considered feasible for this region. The present study reports a rare case of an ITF neurofibroma arising from the inferior alveolar nerve. The 27-year-old male patient presented with numbness of the right jaw. A radiological examination revealed a large mass occupying the ITF, from the mandible to the foramen ovale, originating from the inferior alveolar nerve in the mandible. The tumor was successfully excised via a transtemporal approach followed by a transoral-retromolar approach. A histological examination confirmed the diagnosis of neurofibroma. The present case demonstrates that a combination of the transtemporal and transoral-retromolar approaches may provide wide access to the ITF region.
\end{abstract}

\section{Introduction}

Neurofibroma is usually associated with neurofibromatosis (NF) type 1 , and is among the most common neurogenic tumors of the head and neck region in NF-1 patients. Solitary cases that are not associated with NF-1 are rarely reported. Among these, neurofibromas arising from peripheral divisions of the trigeminal nerve are even rarer (1-6). In the majority of cases, the tumor tends to extend outside the mandible, leading to deformity of the jaw as the initial symptom $(3,7)$. Other common symptoms include pain or paresthesia of the affected division of the trigeminal nerve.

The excision of tumors located in the infratemporal fossa (ITF) is challenging, as such lesions are surrounded by the skull base, mandible, lateral and medial pterygoid plate,

Correspondence to: Dr Takuro Inoue, Department of Neurosurgery, Subarukai Kotoh Kinen Hospital, 2-1 Hiramatsu-cho, Higashiohmi, Shiga 527-0134, Japan

E-mail: takuro39@gmail.com

Key words: neurofibroma, trigeminal nerve, inferior alveolar nerve, infratemporal fossa, surgical approach maxillary sinus and parapharyngeal structures. A number of approaches, including the transtemporal-transzygomatic, transzygomatic-transmandibular, transmaxillary, posterior high-cervical and transoral approaches, may be applied, depending on the tumor location (8-16). This study presents a rare case of a trigeminal neurofibroma arising from the inferior alveolar nerve, which was successfully excised via a combination of the transtemporal and transoral-retromolar approaches, as well as a discussion of these two approaches.

\section{Case report}

A 27-year-old man presented in June 2015 with progressive numbness of the right jaw, and was diagnosed with an ITF tumor at another hospital. The only neurological finding was paresthesia in the area innervated by the mental nerve of the trigeminal mandibular division. The patient's taste sensation was normal, and the motor function of the trigeminal nerve was intact. There was no inflammatory reaction in the parapharyngeal area or family history of NF. A computed tomography (CT) scan revealed a non-enhanced mass in the infratemporal region, with an enlarged mandibular canal and foramen ovale, suggesting a neurogenic tumor along the third branch of the trigeminal nerve (Fig. 1A and B). A 3D-CT scan clearly depicted the mass located within the mandible, occupying the ITF and widening the foramen ovale. The internal carotid artery was displaced posteriorly, and the maxillary artery ran along the anterior surface of the tumor (Fig. 1C and D). A magnetic resonance imaging (MRI) scan revealed a large mass in the ITF, extending from the intramandibular region to the foramen ovale. The mass exhibited no enhancement following administration of contrast medium (Fig. 2).

As the patient's symptoms progressed, a staged surgical excision was considered. A transtemporal approach was employed, which involved retraction of the temporal lobe extradurally, followed by drilling around the foramen ovale. Following removal of the upper third of the tumor, the remaining tumor was removed via a transoral-retromolar approach (Fig. 3). The tumor was soft and fibrous, without a capsule, which was consistent with the typical findings of a neurofibroma. As the tumor had originated from the inferior alveolar nerve, preservation of this nerve was not possible. However, the lingual nerve, which was identified on the anterior surface of the tumor, was completely 

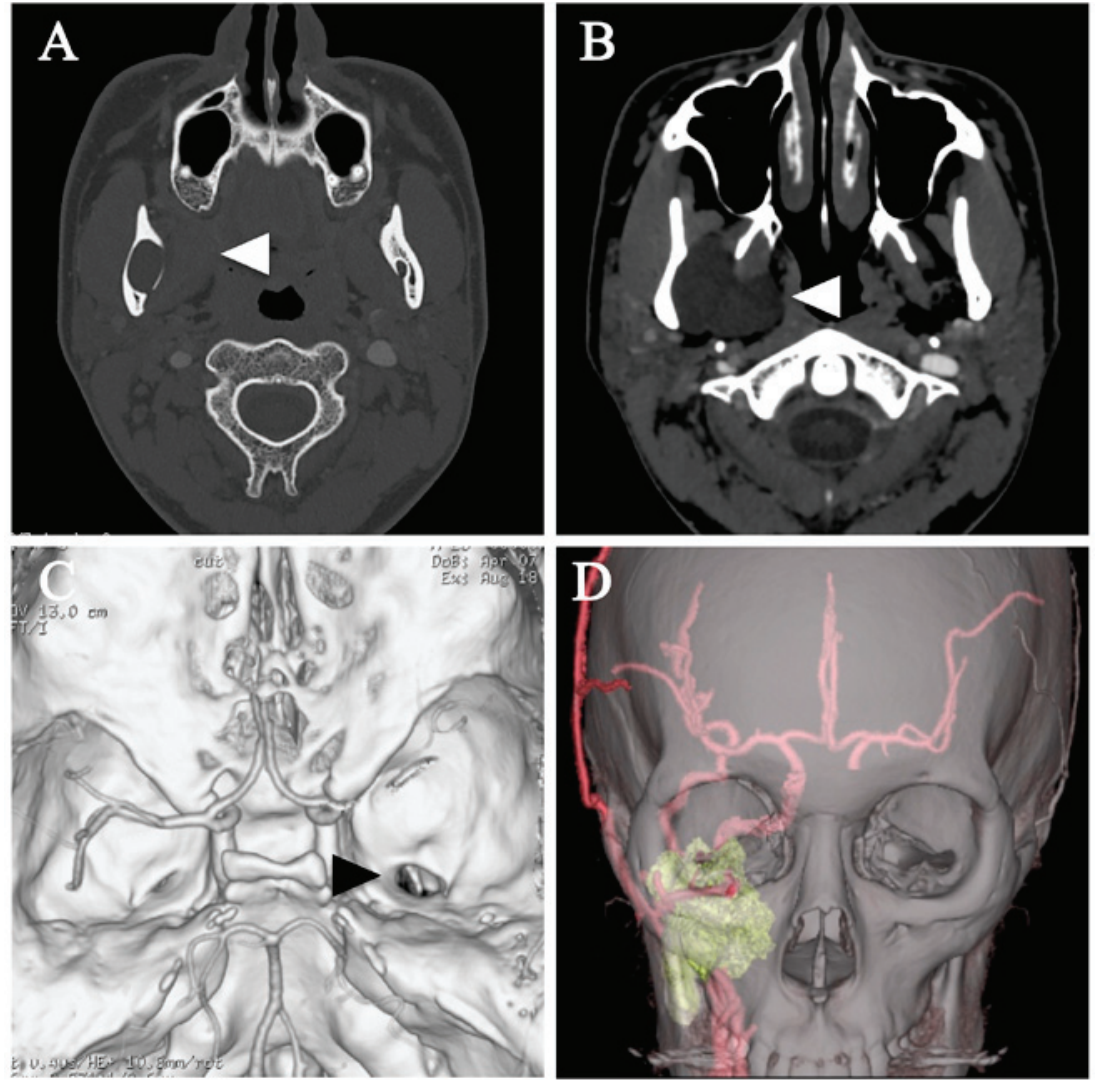

Figure 1. Preoperative computed tomography (CT) images. (A) Bone window CT showing the enlarged mandibular canal (white arrowhead). (B) The tumor appears as a non-enhanced, low-density mass (white arrowhead). (C) Three-dimensional (3D) CT scan of the skull base showing the enlarged foramen ovale (black arrowhead). (D) A 3D-CT scan depicts the tumor location (green) and adjacent vessels (red). The internal carotid artery was located behind the tumor
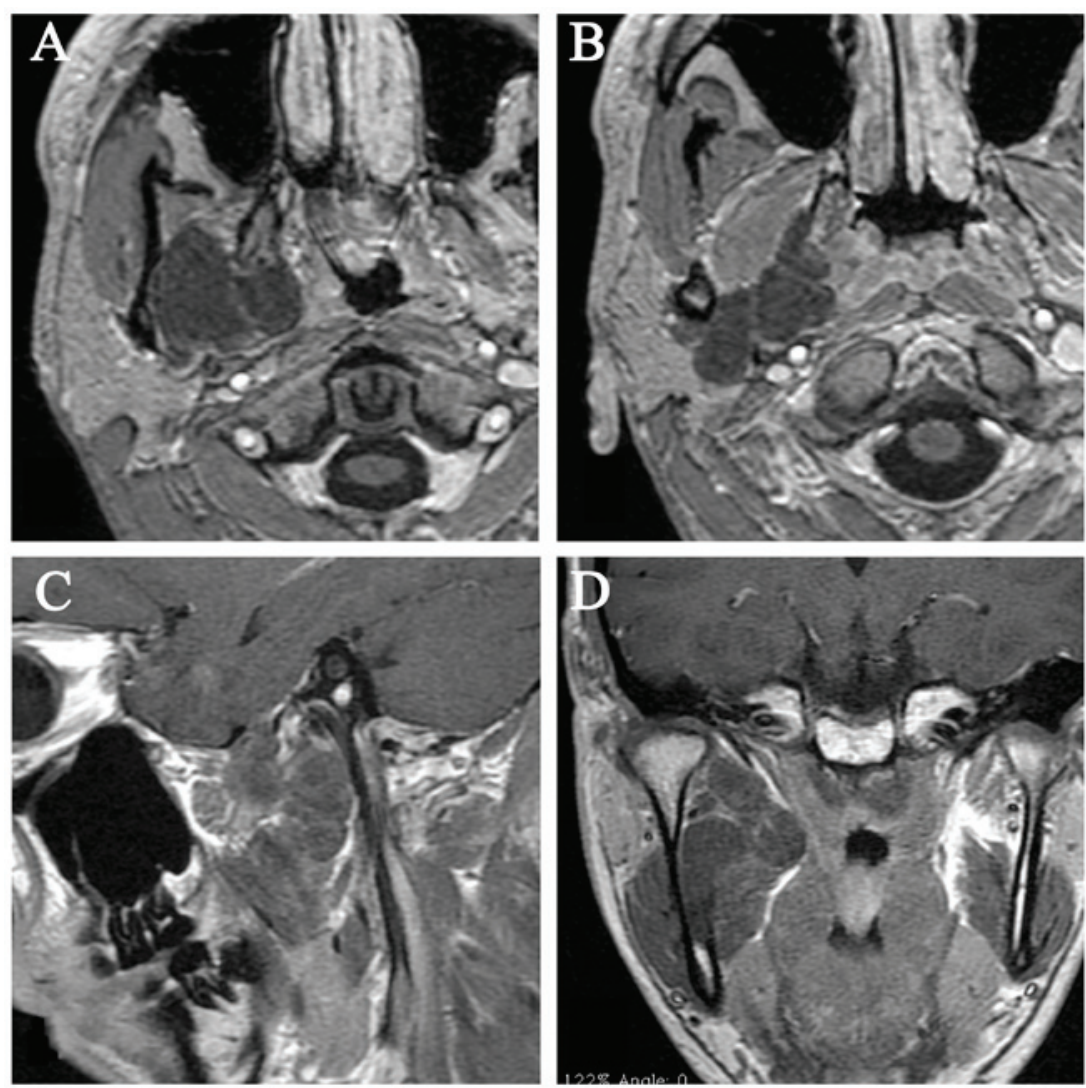

Figure 2. Preoperative magnetic resonance imaging (MRI). (A and B) The axial view of contrast-enhanced MRI (T1-spoiled gradient recalled image) revealed that the mass extended from the infratemporal fossa to the styloid process on the right side. (C) The sagittal view revealed that the mass extended posteriorly around the jugular foramen and the styloid process. (D) The coronal view revealed that the tumor was growing into the mandibular body on the right. 

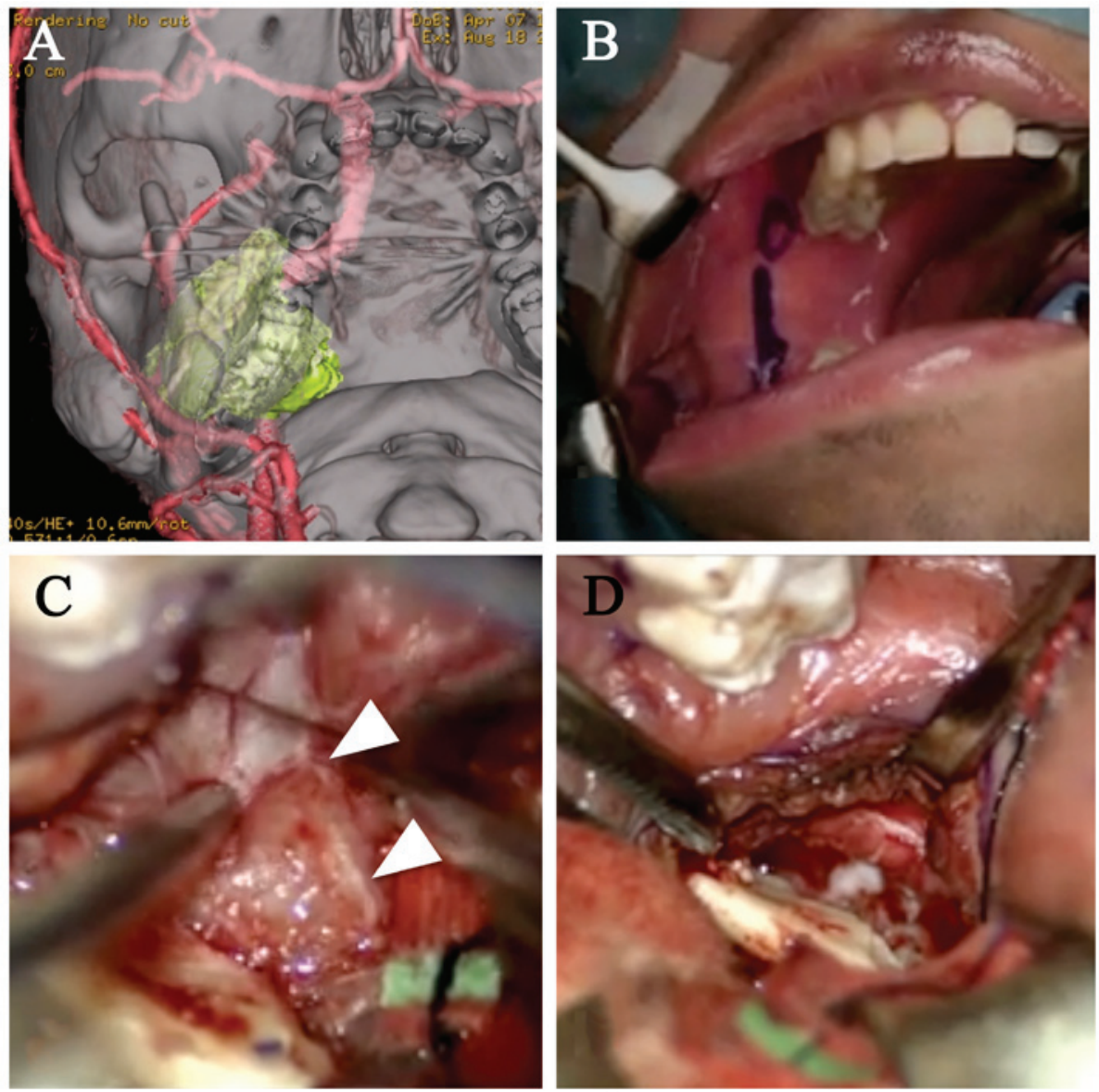

Figure 3. A three-dimensional (3D)-computed tomography (CT) image and operative views. (A) Preoperative 3D-CT view of the intraoral approach. The tumor appeared to be accessible through this approach. (B) A mucosal incision was performed in the transoral-retromolar approach. The small circle on the mucosa indicates the papilla of the parotid duct. (C) The lingual nerve was identified at the anterior medial surface of the tumor (arrowheads). (D) View after complete removal of the tumor. The tumor cavity appeared to be extending to the jugular foramen.

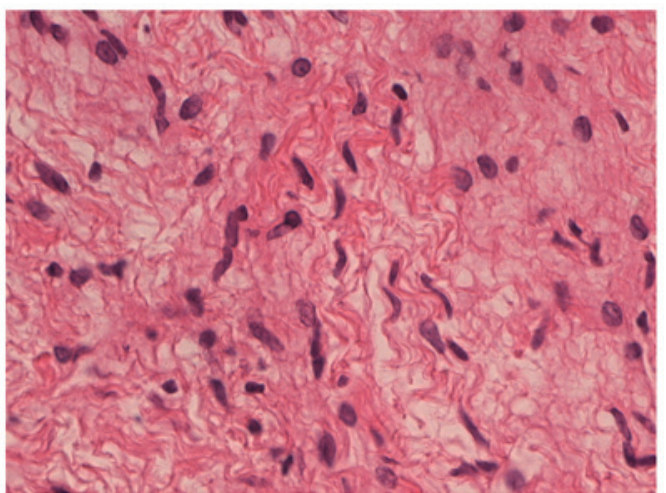

Figure 4. On histological examination, the tumor included a myxoid stroma and cells with serpiginous nuclei, suggesting a neurofibroma. Hematoxylin and eosin staining, magnification, $\mathrm{x} 400$.

preserved. Using Doppler ultrasound and intraoperative navigation, the course of the internal carotid artery was identified as running immediately behind the tumor and then entering into the carotid canal.

Histological examination revealed that the tumor cells were situated in a loose, delicate and myxoid stroma, and had wavy or serpiginous nuclei, suggesting a neurofibroma (Fig. 4). Immunohistochemical examination revealed that
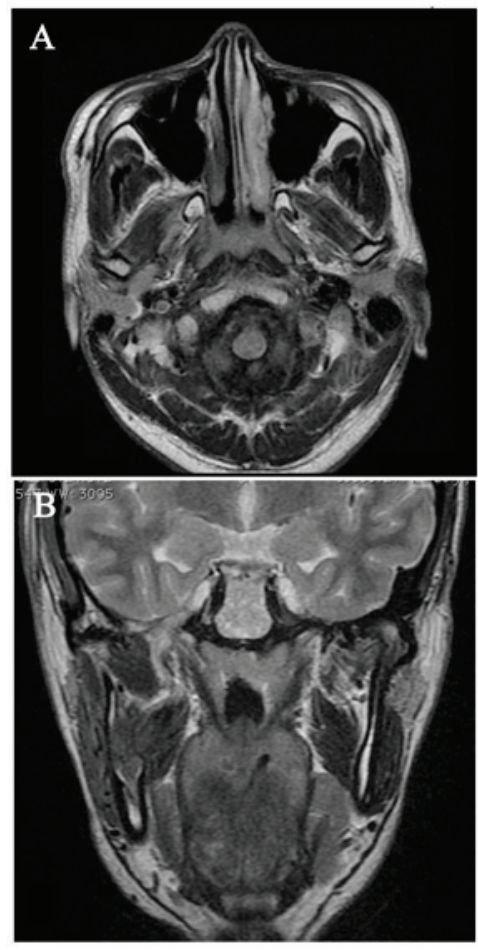

Figure 5. Postoperative magnetic resonance imaging (MRI). (A and B) Axial and coronal views of the postoperative MRI showing total removal of the tumor. The lateral and medial pterygoid muscles have returned to their normal position. 
the lesion contained numerous S-100 protein-positive cells. Immunoreactivity for neurofilament protein was observed in the scattered axons. There was no immunoreactivity for epithelial membrane antigen. As the patient had no other neurofibromas, the diagnosis was established as solitary neurofibroma of the inferior alveolar nerve in the mandible.

The patient's neurological symptom did not subside; the numbness of the right jaw, which was present prior to surgery, persisted postoperatively. No recurrence was observed on an MRI scan 12 months after the surgery (Fig. 5). The last follow-up visit was in June 2016. Written informed consent was obtained from the patient regarding the publication of this case report and associated images.

\section{Discussion}

Neurofibroma is a benign tumor that arises from neurons and perineural cells (5). It is most commonly associated with NF-1, as multiple tumors may arise from peripheral nerves in NF-1 patients (1). On the contrary, solitary neurofibromas arising from a division of the trigeminal nerve are rarely reported (1-7). Neurofibromas arising from the inferior alveolar nerve usually present with deformity of the jaw, due to outgrowth of the mandible, as well as sensory disturbance of the affected nerve (1-3). Ellis et al summarized intraosseous benign neural sheath neoplasms of the jaw and described the characteristics of neurofibromas and neurinomas in this region (7). Solitary neurofibromas are reported to be slow-growing tumors; however, an unencapsulated neurofibroma has a greater probability of recurrence compared with an encapsulated neurinoma. Therefore, surgical resection plays an important role in the treatment of solitary neurofibromas in this region.

The ITF is among the most challenging regions to access $(8,9,11,13)$. In addition, in cases with significant tumor extension, more than one approach may be required to remove the tumor. In the present case, the tumor extended from the body of the mandible to the foramen ovale, and was difficult to expose via a single approach. Thus, two approaches were used: A transtemporal approach for the upper part of the tumor, and a transoral-retromolar approach for the lower part of the tumor. The transtemporal approach is a transcranial extradural approach to the region (8) that provides a wide operative field around the foramen ovale, where most divisions of the mandibular nerve can be observed. However, the width of the operative field depends on the area of the middle fossa that can be drilled away. Furthermore, although extensive drilling of the middle fossa and removal of the zygomatic arch provide easy access to the medial and deeper areas of the ITF, exposing the lateral part of the ITF, just inside the mandibular ramus, requires excessive retraction of the temporal lobe. In addition, even with extensive posterior bone drilling, the temporomandibular joint limits the posterior surgical exposure around the carotid canal and styloid process. Therefore, a transtemporal approach is not suitable for the removal of masses located in the lateral and posterior parts of the ITF.

By contrast, the region difficult to reach via the aforementioned transtemporal approach may be reached easily via a transoral-retromolar approach $(12,13)$. The gingival incision of the oral mucosa may be performed in the retromolar trigone, allowing direct exposure around the mandibular ramus, where the inferior alveolar nerve enters into the mandibular canal. Then, following the inferior alveolar nerve proximally, dissection of the connective tissues between the medial and lateral pterygoid muscles leads to the posterior trunk of the mandibular nerve, from where the lingual nerve branches just below the lateral pterygoid muscle. In contrast to the limitation of a surgical corridor formed by the window of bone drilling in the transtemporal approach, the entry from the oral mucosa allows flexible changes in the direction of the surgeon's view. In the present case, the tumor around the jugular foramen could not be reached via the transtemporal approach, but was easily identified and removed via the transoral-retromolar approach.

The transzygomatic-transmandibular approach is another useful approach for accessing the $\operatorname{ITF}(9,10)$. However, the facial nerve is at risk while dissecting around the parotid gland to expose the lateral ramus of the mandible. In addition, reconstruction of the mandible and temporomandibular joint is a major concern following tumor removal. The use of the transoral-retromolar approach is free from the risk of facial nerve injury or the necessity for reconstruction of the mandible. In addition, accessing the ITF and the subsequent closure are fast and simple, as only the gingival incision is required to create the corridor to the ITF (13). However, the risk of infection may be relatively high compared with other approaches due to the transoral route. Therefore, in cases involving a tumor of the ITF extending intradurally in the middle fossa, the transoral-retromolar approach must be applied following careful consideration.

\section{References}

1. Apostolidis C, Anterriotis D, Rapidis AD and Angelopoulos AP: Solitary intraosseous neurofibroma of the inferior alveolar nerve: Report of a case. J Oral Maxillofac Surg 59: 232-235, 2001.

2. Jangam SS, Ingole SN, Deshpande MD and Ranadive PA: Solitary intraosseous neurofibroma: Report of a unique case. Contemp Clin Dent 5: 561-563, 2014.

3. Polak M, Polak G, Brocheriou C and Vigneul J: Solitary neurofibroma of the mandible: Case report and review of the literature. J Oral Maxillofac Surg 47: 65-68, 1989.

4. da Rosa MR, Ribeiro AL, de Menezes SA, Pinheiro JJ and Alves-Junior SM: Solitary giant neurofibroma of the mental nerve: A trauma-related lesion? J Craniofac Surg 24: e247-e251, 2013.

5. Wang HM, Hsu YC, Lee KW, Chiang FY and Kuo WR: Neurofibroma of the lingual nerve: A case report. Kaohsiung J Med Sci 22: 461-464, 2006.

6. Cartellieri M and Swoboda H: Neurofibroma of the auriculotemporal nerve. Eur Arch Otorhinolaryngol 257: 396-398, 2000.

7. Ellis GL, Abrams AM and Melrose RJ: Intraosseous benign neural sheath neoplasms of the jaws. Report of seven new cases and review of the literature. Oral Surg Oral Med Oral Pathol 44: 731-743, 1977.

8. Terasaka S, Sawamura Y, Goto S and Fukushima T: A lateral transzygomatic-transtemporal approach to the infratemporal fossa: Technical note for mobilization of the second and third branches of the trigeminal nerve. Skull Base Surg 9: 277-287, 1999.

9. Timoshenko AP, Asanau A, Gavid M, Colin V, Martin C and Prades JM: Preauricular transmandibular and transzygomatic approach for tumors of the infratemporal fossa revisited. ORL J Otorhinolaryngol Relat Spec 75: 250-255, 2013.

10. Chatni SS, Sharan R, Patel D, Iyer S, Tiwari RM and Kuriakose MA: Transmandibular approach for excision of maxillary sinus tumors extending to pterygopalatine and infratemporal fossae. Oral Oncol 45: 720-726, 2009.

11. Liu JK, Sameshima T, Gottfried ON, Couldwell WT and Fukushima T: The combined transmastoid retro- and infralabyrinthine transjugular transcondylar transtubercular high cervical approach for resection of glomus jugulare tumors. Neurosurgery 59 (1 Suppl 1): ONS115-125, 2006. 
12. Cobzeanu BM, Popescu E, Costan VV, Ungureanu D and Cobzeanu MD: Retromolar trigone-oropharynx junction maligns tumor surgery: Transmandibular versus oral approach. Rev Med Chir Soc Med Nat Iasi 119: 119-126, 2015.

13. Scheller K, Eckert AW and Scheller C: Transoral, retromolar, para-tonsillar approach to the styloid process in 6 patients with Eagle's syndrome. Med Oral Patol Oral Cir Bucal 19: e61-e66, 2014.

14. El-Sayed I, Pletcher S, Russell M, McDermott M and Parsa A: Endoscopic anterior maxillotomy: Infratemporal fossa via transnasal approach. Laryngoscope 121: 694-698, 2011.
15. Carrau RL, Prevedello DM, de Lara D, Durmus K and Ozer E: Combined transoral robotic surgery and endoscopic endonasal approach for the resection of extensive malignancies of the skull base. Head Neck 35: E351-E358, 2013.

16. Satar B, Yazar F, Ceyhan A, Arslan HH and Aydin S: Analysis of jugular foramen exposure in the fallopian bridge technique. Skull Base 19: 203-207, 2009. 